\title{
NUEVA CLASIFICACIÓN INFRAGENÉRICA DE AZORELLA (APIACEAE, AZORELLOIDEAE) Y SINOPSIS DEL SUBGÉNERO ANDINAE
}

\author{
Martina Fernández \& Carolina I. Calviño
}

\begin{abstract}
Instituto de Investigaciones en Biodiversidad y Medioambiente (INIBIOMA), Universidad Nacional del ComahueCONICET, Quintral 1250, 8400 Bariloche, Río Negro, Argentina; mfernandez@comahue-conicet.gob.ar ccalvino@comahue-conicet.gob.ar (autoras corresponsales).
\end{abstract}

\begin{abstract}
Fernández, M. \& C. I. Calviño. 2019. New infrageneric classification of Azorella (Apiaceae, Azorelloideae) and synopsis of subgenus Andinae. Darwiniana, nueva serie 7(2): 289-304.

A recent circunscription of Azorella reduced the genera Schizeilema, Stilbocarpa, Huanaca, Laretia, and Mulinum to its synonymy. Based on the integration of morphological studies, taxonomic revisions and molecular phylogenies, we propose here the division of Azorella into two subgenera: Azorella and Andinae. These two groups are strongly supported in all phylogenies, and represent the first divergence event in the genus, resulting in independent evolutionary histories, so it is important to highlight these differences. The subgenus Azorella includes 23 herbaceous species with a distribution in austral regions of the Southern Hemisphere, including Patagonia, Australia, New Zealand and the subantarctic islands; it comprises the formerly recognized genera Huanaca, Schizeilema, Stilbocarpa, and the three species of Azorella Sect. Azorella. On the other hand, the subgenus Andinae includes 35 woody species mainly of South American distribution and in high altitude regions of the Andes and Patagonia; it includes the remaining species traditionally treated in Azorella, plus Mulinum and Laretia. A morphological description of Azorella, as circuncribed today, and a key to differentiate the two subgenera are provided for the first time. Given the current thorough and integrated knowledge of the morphology, anatomy, and geographic distribution of all species of subgenus Andinae, a key is provided for all its species and varieties, as well as lists of synonyms and taxonomic observations. In addition, we publish 6 new combinations, and correct nomenclatural errors.
\end{abstract}

Keywords. Andes; Huanaca; Laretia; Mulinum; nomenclature; Schizeilema; Southern Hemisphere; Stilbocarpa; Umbelliferae.

Resumen. Fernández, M. \& C. I. Calviño. 2019. Nueva clasificación infragenérica de Azorella (Apiaceae, Azorelloideae) y sinopsis del subgénero Andinae. Darwiniana, nueva serie 7(2): 289-304.

Una reciente circunscripción de Azorella reduce los géneros Schizeilema, Stilbocarpa, Huanaca, Laretia, y Mulinum a su sinonimia. En base a la integración de estudios morfológicos, revisiones taxonómicas y filogenias moleculares, se propone la división de Azorella en dos subgéneros: Azorella y Andinae. Estos dos grupos están fuertemente soportados en todas las filogenias y representan el primer evento de divergencia en el género, que resultó en historias evolutivas independientes, por lo que es importante resaltar estas diferencias. El subgénero Azorella incluye 23 especies herbáceas con una distribución en zonas australes del Hemisferio Sur, incluyendo la Patagonia, Australia, Nueva Zelanda e islas subantárticas; reúne a los géneros anteriormente reconocidos Huanaca, Schizeilema y Stilbocarpa y a las tres especies de Azorella Sect. Azorella. Por otro lado, el subgénero Andinae comprende 35 especies con una distribución principalmente sudamericana y dominante en regiones elevadas de los Andes y en la estepa Patagónica; reúne a las restantes especies tradicionalmente tratadas en Azorella, más Mulinum y Laretia. Se aporta por primera vez una descripción morfológica de Azorella, según su circunscripción actual, y una clave para diferenciar a los dos subgéneros. Dado que para todas las especies del subgénero Andinae se dispone de un conocimiento amplio e integrado de la morfología, anatomía y de su distribución geográfica, se proporciona una clave para todas las especies y variedades del subgénero, así como listas de sinónimos y observaciones taxonómicas. Además, se realizan 6 combinaciones nuevas y se corrigen errores nomenclaturales.

Palabras clave. Andes; Hemisferio Sur; Huanaca; Laretia; Mulinum; nomenclatura; Schizeilema; Stilbocarpa; Umbelliferae. 


\section{INTRODUCCIÓN}

Una reciente clasificación [Plunkett \& Nicolas, 2017 (2016 on-line)] considera a los géneros Schizeilema (Hook. f.) Domin, Stilbocarpa (Hook. f.) Decne. \& Planch., Huanaca Cav., Laretia Gillies \& Hook. y Mulinum Pers. (grupo SHALM) bajo un único género: Azorella, con el fin de resaltar el origen común de estos grupos y evitar el uso de taxones polifiléticos. Fernández et al. (2017a), en un trabajo filogenético que se publicó sin conocimiento del de Plunkett \& Nicolas [2017 (2016 on-line)], discutieron y resaltaron la complejidad de circunscribir estos seis géneros en grupos que reflejen monofilia. Todas las filogenias publicadas [Andersson et al., 2006; Nicolas \& Plunkett, 2009, 2012; Plunkett \& Nicolas, 2017 (2016 on-line); Fernández et al., 2017a)] coinciden en que el grupo SHALM se divide en dos grandes linajes que Fernández et al. (2017a) llamaron informalmente Andino-Patagónico y Austral. Allí, se indicó la importancia de nombrar formalmente al linaje Andino-Patagónico, pero no se tomaron decisiones taxonómicas ya que se consideró que no se contaba con información filogenética y morfológica suficientes en el linaje Austral, como para garantizar estabilidad nomenclatural y una correcta evaluación de los linajes a nombrar en la categoría de género. Dado que en la propuesta de Plunkett \& Nicolas (2017 (2016 on-line)) ya se reunió al grupo SHALM en un único género y se realizaron los cambios nomenclaturales correspondientes, por el momento, lo más conservador y consistente con el objetivo de reflejar grupos monofiléticos en las clasificaciones, es seguir esta propuesta.

En la circunscripción de Azorella de Plunkett \& Nicolas [2017 (2016 on-line)] se dividió al género en 10 secciones, que corresponden a grupos monofiléticos o a especies con una posición incierta en las filogenias del grupo. Sin embargo, los dos linajes principales del género (i.e., los linajes Austral y Andino-Patagónico) no fueron nombrados formalmente. Estos grupos están fuertemente soportados en todas las filogenias [Andersson et al., 2006; Nicolas \& Plunkett, 2009, 2012; Plunkett \& Nicolas, 2017 (2016 on-line); Fernández et al., 2017a] y representan el primer evento de divergencia en el género, que resultó en historias evolutivas independientes (Fernández et al., 2017a), por lo que es importante resaltar estas diferencias.
En el presente trabajo se provee por primera vez una descripción morfológica del género Azorella, según la reciente circunscripción de Plunkett \& Nicolas [2017 (2016 on-line)]. Se propone una nueva clasificación infragenérica que reconozca a los linajes Austral y AndinoPatagónico como subgéneros de Azorella: Azorella y Andinae. Se incluyen sus diagnosis y clave para diferenciarlos. Además, se presenta una lista de las especies aceptadas en cada subgénero, incluyendo su distribución geográfica, revisiones taxonómicas o referencias a descripciones morfológicas e iconografía (Tabla 1). Todas las especies del subgénero Andinae fueron estudiadas filogenéticamente [Andersson et al., 2006; Nicolas \& Plunkett, 2009, 2012; Plunkett \& Nicolas, 2017 (2016 on-line); Fernández et al., 2017a], morfológica y taxonómicamente (Martínez, 1989, 1993, 1995; Calviño et al., 2016; Fernández et al., 2016a, b, 2017b, c, en prensa). Sin embargo, hasta el momento el conocimiento sobre la morfología y taxonomía, así como la posición filogenética de la mayoría de las especies del subgénero Azorella es fragmentario y dispar (ver discusión en Fernández et al., 2017a). Por lo tanto, en este trabajo y para este subgénero, solamente se listan los nombres aceptados para todas las especies. Por el contrario, para el subgénero Andinae, se aporta adicionalmente una clave para la identificación de sus especies y variedades y se lista la sinonimia en cada caso.

\section{MATERIALES Y MÉTODOS}

El material estudiado corresponde al analizado para la subfamilia Azorelloideae para Flora Argentina y en las revisiones taxonómicas y nomenclaturales de Azorella, Mulinum y Laretia (Martínez, 1989, 1993; Calviño et al., 2016; Fernández et al., 2016b, 2017a, b, c; Fernández, M., Martínez, S. \& C. I. Calviño, en prensa) y proviene de los siguientes herbarios: $\mathrm{B}, \mathrm{BA}, \mathrm{BAA}, \mathrm{BAB}, \mathrm{BC}$, BCRU, BM, COL, CONC, CORD, E, F, FR, G, GH, GOET, HAL, JE, K, KW, LA, LAU, LD, LIL, LP, LPB, LY, M, MA, MCNS, MERL, MO, MPU, NY, P, PR, PRC, QCNE, S, SGO, SI, TCD, UC, UPS, US, VEN, W (Thiers, 2019). 
También se analizaron las siguientes revisiones taxonómicas o florísticas que incluyen claves para el reconocimiento de las especies, listas de sinónimos, descripciones morfológicas, observaciones sobre afinidades morfológicas entre las especies, y/o mapas de distribución de Huanaca (Mathias \& Constance, 1971), Schizeilema y Stilbocarpa (Allan, 1961; Jacobs \& Pickard, 1981; PlantNET, 2019).

Las nuevas combinaciones establecidas en este trabajo siguen el Código Internacional de Nomenclatura Botánica (ICN; Turland et al., 2018). En aquellos casos para los cuales fue necesario actualizar la sinonimia, se estudió el material tipo disponible o fotografías de los mismos y los protólogos correspondientes.

\section{TRATAMIENTO TAXONÓMICO}

En base a la integración de estudios morfológicos, revisiones taxonómicas y filogenias moleculares, el género Azorella se clasifica en dos subgéneros, Andinae y Azorella, que reconoce a los linajes que Fernández et al. (2017a) llamaron informalmente Andino-Patagónico y Austral, respectivamente. Esta nueva clasificación es compatible con la división en secciones de Plunkett \& Nicolas [2017 (2016 on-line)]. De esta manera, el subgénero Andinae incluye las secciones: Spinosae S. Martínez, Laretia (Gillies \& Hook.) G. M. Plunkett \& A. N. Nicolas, Pectophytum (Kunth) Kuntze, Cirrhosae Hauman, y Glabratae Hauman, y el subgénero Azorella incluye las secciones: Azorella, Schizeilema (Hook.f.) Benth \& Hook.f., Huanaca (Cav.) Kuntze, Ranunculus G. M. Plunkett \& A. N. Nicolas, y Stilbocarpa (Hook.f.) G. M. Plunkett \& A. N. Nicolas.

El subgénero Andinae es principalmente sudamericano y ecológicamente importante por su dominancia en regiones elevadas de los Andes y en la estepa Patagónica; comprende a 35 especies, tradicionalmente incluidas en los géneros Mulinum, Laretia y Azorella. Su grupo hermano el subgénero Azorella se distribuye en zonas australes del Hemisferio Sur, incluyendo la Patagonia, Australia, Nueva Zelanda e islas subantárticas; comprende 23 especies, previamente pertenecientes a los géneros Huanaca, Schizeilema, Stilbocarpa y a Azorella sección Azorella (3 especies, incluyendo la especie tipo del género). Ambos subgéneros son fácilmente diferenciables por el hábito herbáceo en las especies del subgénero Azorella y leñoso en Andinae, siendo esta última una característica sinapomórfica de este linaje (Fernández et al., 2017a).

Las especies aquí aceptadas y sus sinónimos se basan en revisiones taxonómicas o florísticas disponibles por décadas (Allan, 1961; Mathias \& Constance, 1971; Constance, 1988; Martínez, 1989) y en revisiones recientes donde se re-evaluó la delimitación de las especies (Calviño et al., 2016; Fernández et al., 2016b, 2017b, c). Las decisiones taxonómicas por tanto difieren parcialmente de las de Plunkett \& Nicolas [2017 (2016 on-line)]; Tabla 1. Como resultado se aceptan 58 especies en el género Azorella, se establecen 6 combinaciones nuevas y se corrigen errores nomenclaturales.

Azorella Lam., Encycl. Meth., Bot. 1: 344. 1783. ESPECIE TIPO: Azorella filamentosa Lam.

Plantas olorosas, frecuentemente resinosas, perennes, ramosas, espinescentes o no, herbáceas erectas a rastreras o leñosas formando cojines o matas. Hojas glabras o pubescentes; sésiles o pecioladas; estípulas ausentes o presentes y parcialmente adnatas a la vaina foliar. Inflorescencia de umbelas simples o raro compuestas, involucradas, uni- a plurifloras, subsésiles o pedunculadas; terminales y solitarias o acompañadas por una o varias umbelas axilares. Flores subsésiles o pediceladas; sépalos reducidos a dientes, evidentes a inconspicuos, persistentes a la madurez; pétalos blancos, púrpuras, verdosos o amarillentos; ápice plano o débilmente inflexo, caedizos a la madurez; estambres más largos que los estilos, o ausentes o reducidos a estaminodios hialinos en las flores pistiladas; estilos 2 (3-4 en $A$. polaris), persistentes o no en el fruto maduro; estilopodio deprimido a cónico. Fruto esquizocarpo terete o comprimido dorsalmente, o muy raro drupáceo subgloboso, glabro o, excepcionalmente, escamoso, alado o áptero, canales secretores poco a muy desarrollados, uno por costilla; endocarpio leñoso; carpóforo ausente o presente, libre o persistentemente unido a uno de los mericarpios, cuando libre entero o bífido hasta bipartido. 


\section{Clave de los subgéneros de Azorella}

1. Plantas herbáceas. Hojas con estípulas, o sin ellas, entonces vaina foliar profundamente laciniada Azorella subgen. Azorella

1. Plantas leñosas. Hojas sin estípulas; vaina foliar entera, con cilias o sin ellas Azorella subgen. Andinae

\section{Azorella Lam. subgen. Azorella}

Chamitis Gaertn., Fruct. Sem. Pl. 1: 94. t. 22. 1788. ESPECIE TIPO: Chamitis integrifolia Gaertn. [=Azorella filamentosa Lam.].

Huanaca Cav., Icon. 6: 18. 1801. ESPECIE TIPO: Huanaca acaulis Cav. [=Azorella acaulis (Cav.) Drude].

Stilbocarpa (Hook.f.) Decne. \& Planch. Rev. Hort. 3: 105. 1854. ESPECIE TIPO: Stilbocarpa polaris (Hombr. \& Jacq.) A. Gray [=Azorella polaris (Hombr. \& Jacq.) G. M. Plunkett \& A. N. Nicolas].

Trisciadium Phil. Anales Univ. Chile. 1: 61. 1861. ESPECIE TIPO: Trisciadium andinum Phil. [=Azorella andina (Phil.) Drude].

Lechleria Phil., Linnaea 28: 654. 1858. ESPECIE TIPO: Lechleria palmata Phil. [= Azorella acaulis (Cav.) Drude].

Pozoa Lag. subgen. Schizeilema Hook.f. Fl. Antarct. 15. 1844. Schizeilema (Hook.f.) Domin, Bot. Jahrb. Syst. 40: 573. 1908. ESPECIE TIPO: Pozoa reniformis Hook.f. [=Azorella schizeilema G.M. Plunkett \& A.N. Nicolas].

Kirkophytum (Harms) Allan, Fl. New Zealand 1: 431. 1961. ESPECIE TIPO: Kirkophytum lyallii (Armstr.) Allan [=Azorella lyallii (Armstr.) G.M. Plunkett \& A.N. Nicolas].

Plantas herbáceas, erectas a rastreras, a veces formando cojines.

Incluye 23 especies, Tabla 1 (especies numeradas desde el 1 al 23).

Distribución geográfica. Subgénero de zonas australes del hemisferio sur, por debajo de los $30^{\circ} \mathrm{S}$. Habita el sur de los Andes templados de Chile y Argentina, Nueva Zelanda, Australia (estados New South West y Victoria) y las islas subantárticas Antipodes, Auckland, Campbell, Crozet, Heard, Kerguelen, Macquarias, Malvinas, Marion, McDonald, Norte, Prince Edward, Snares, Stewart y Sur.
Referencias. Véase Allan (1961), Mathias \& Constance (1971), Jacobs \& Pickard (1981), Martínez (1989), Constance (1988), y PlantNET (2019) para revisiones taxonómicas o florísticas donde se encuentran descripciones morfológicas e iconografía para todas las especies (Tabla 1).

Azorella Lam. subgen. Andinae Mar. Fernández \& C.I. Calviño, subgen. nov. ESPECIE TIPO: Azorella compacta Phil.

Fragosa Ruiz \& Pav., Fl. Peruv. Prodr. 43, t. 34. 1794. ESPECIE TIPO: Fragosa multifida Ruiz $\&$ Pav. [=Azorella multifida (Ruiz \& Pav.) Pers.]. Mulinum Pers., Syn. P1. 1: 309. 1805. ESPECIE TIPO: Mulinum spinosum (Cav.) Pers. [=Azorella prolifera (Cav.) G.M. Plunkett \& A.N. Nicolas].

Pectophytum Kunth, Nov. Gen. Sp. 5: 28-29. 1821. ESPECIE TIPO: Pectophytum pedunculare Kunth, nom. illeg. $[=$ Bolax pedunculatus Spreng. $=$ Azorella pedunculata (Spreng.) Mathias \& Constance].

Laretia Gillies \& Hook., Bot. Misc. 1: 329, t. 65. 1830. ESPECIE TIPO: Laretia acaulis (Cav.) Gillies \& Hook. [=Azorella ruizii G.M. Plunkett \& A.N. Nicolas].

Apleura Phil. Linnaea 33: 89. 1864. ESPECIE TIPO: Apleura nucamentacea Phil. [=Azorella monantha Clos].

Azorellopsis H. Wolff, Repert. Spec. Nov. Regni Veg. 19:312. 1924. ESPECIE TIPO: Azorellopsis trisecta $\mathrm{H}$. Wolff. [=Azorella trisecta (H. Wolff) Mar. Fernández \& C.I. Calviño].

Woody plants that form hemispherical or flat cushions either loose or compact, or small shrubs (mats).

Plantas leñosas que forman cojines hemisféricos o chatos, o subarbustos bajos (matas).

Incluye 35 especies, Tabla 1 (especies numeradas desde el 24 al 58). 
Distribución geográfica y hábitat. Subgénero principalmente sudamericano, característico de las cordilleras andinas y de la estepa patagónica, desde Costa Rica hasta Tierra del Fuego e islas subantárticas (Crozet, Heard, Kerguelen, Macquarias, Malvinas, Marion, McDonald y Prince Edward). Se extiende desde el nivel del mar hasta los $5200 \mathrm{~m}$ s.m, dependiendo de la latitud. Las especies crecen en regiones de clima templado, en ambientes abiertos, variados, que van desde estepas áridas en zonas secas con terrenos en general arenosos, pedregosos y/o rocosos, hasta zonas húmedas que incluyen bordes de mallines y vegas, y orillas de ríos y lagos, en las provincias fitogeográficas del Páramo, Puneña, Altoandina, Patagónica e Insular (Cabrera, 1971; Cabrera \& Willink, 1980).

Referencias. Véase Martínez (1989), Zech (1997), Calviño et al. (2016), y Fernández et al. $(2016 b ; 2017 \mathrm{c})$ para revisiones taxonómicas donde se encuentran descripciones morfológicas e iconografía para todas las especies.

\section{Clave de especies de Azorella subgen. Andinae}

1. Frutos alados

2. Hojas 3-sectas (raro 3-partidas)

3(2). Ápice de los segmentos foliares no punzante y terminados en un cilio fino, largo y blanco, caedizo. Brácteas del involucro formando una cúpula densamente lanosa en el interior. Pétalos espatulados, marcadamente angostados en la base

3. Ápice de los segmentos foliares punzante y mucronado, espinuloso o espinoso. Brácteas del involucro formando una cúpula glabra en el interior. Pétalos nunca espatulados, no angostados en la base

4(3). Cojines chatos y compactos. Segmentos foliares no articulados en su inserción, algunos articulados. Hojas de (5-)7-10(-11) $\mathrm{mm}$ de largo. Lámina foliar de (2-)3-4(-6) $\mathrm{mm}$ de ancho. Pecíolo de 1-3(-4) $\mathrm{mm}$ de largo. Pedúnculos de (6-)8-11(-16) mm de largo A. hallei

4. Matas bajas y laxas. Segmentos foliares articulados en su inserción. Hojas de (9-)11-14(-15) mm de largo. Lámina foliar de 6-8(-9) mm de ancho. Pecíolo de (2-)3-6(-7) mm de largo. Pedúnculos de (11-)19-30(-39) mm de largo

5(3). Segmento foliar central de 1-4 mm de largo, relación largo/ancho menor a 2(-2,5), ápice mucronado o espinuloso 6

5. Segmento foliar central de (3,5-)4-19(-23) $\mathrm{mm}$ de largo, relación largo/ancho mayor o igual a $(1,5) 2$, ápice espinoso 9

6(5). Carpóforo libre. Matas bajas y laxas hasta de $50 \mathrm{~cm}$ de altura y $20 \mathrm{~cm}$ de diámetro. Plantas androdioicas, pedicelos florales de 3-4,5 $\mathrm{mm}$ de largo A. echegarayi 6. Carpóforo persistentemente unido a uno de los mericarpios. Cojines hemisféricos y laxos, o chatos y compactos, hasta de $20 \mathrm{~cm}$ de altura y $40 \mathrm{~cm}$ de diámetro. Plantas andromonoicas, si androdioicas, pedicelos florales de 2-3 mm de largo

7(6). Cojines androdioicos, hemisféricos y laxos. Hojas crasas. Segmentos foliares de anchamente ovados a muy anchamente ovados (relación largo/ancho menor a 1,4). Salta, Argentina, y en las regiones I a III, Chile A. trisecta 7. Cojines andromonoicos, chatos y compactos. Hojas no crasas. Segmentos foliares de angostamente ovados a ovados (relación largo/ancho mayor a 1,5) o de triangulares a anchamente triangulares. Desde Mendoza al sur, Argentina, y en regiones limítrofes de Chile

8(7). Hojas de (4-)6-8(-10) x (2-)3-4(-9) mm, glabras o a veces pubescentes en la cara adaxial. Segmentos foliares de angostamente ovados a ovados. Fruto de (2-)3-4(-5) $\mathrm{mm}$ de ancho, alas iguales o más angostas que el cuerpo del fruto, de 0,5-1 mm de ancho. Mendoza y Neuquén, Argentina, y en las regiones RM y VII, Chile A. albovaginata 8. Hojas de 8-13(-15) x 4-6(-7) mm, siempre glabras. Segmentos foliares de triangulares a anchamente triangulares. Fruto de (3-)5-6(-7) mm de ancho, alas más anchas que el cuerpo del fruto, de 1-3 mm de ancho. Chubut y Santa Cruz, Argentina y en la región XII, Chile 
9(5). Pedúnculos de 11-43(-50) mm de largo. Cojines hemisféricos y laxos. Hojas de (9-)16-43(-51) mm de largo y umbelas que apenas sobrepasan o que sobrepasan netamente el nivel de las hojas .....

9. Pedúnculos de (2-)3-6(-10) mm de largo. Cojines chatos y compactos o matas bajas, irregulares y laxas. Hojas de 17(-20) mm de largo, o mayores pero entonces, umbelas muy por debajo del nivel de las hojas ................. 12 10(9). Hojas glabras, indumento restringido únicamente a la base foliar A. prolifera var. prolifera 10. Hojas pubescentes 11

11(10). Hojas con pocos pelos muy largos y finos y distribuidos irregularmente en la hoja. Chile en la región VI, provincia de Colchagua

A. prolifera var. hirsuta

11. Hojas con abundantes pelos largos y finos cubriendo toda la hoja y dándole una coloración grisácea característica. Chile en la región III, provincia de Huasco

A. prolifera var. zechii

12(9). Segmentos foliares planos. Cojines chatos y compactos y frutos mayores de $5 \mathrm{~mm}$ de ancho A. nivalis 12. Segmentos foliares cilíndricos. Matas bajas y laxas y frutos generalmente menores de $5 \mathrm{~mm}$ de ancho, o si cojines chatos y compactos, los frutos son menores de $4 \mathrm{~mm}$ de ancho

13(12). Hojas de (21-)25-34(-39) x (13-)16-20(-22) mm. Segmento foliar central de (9-)10-14,5(-17) mm de largo, relación largo/ancho mayor a 8

A. triacantha var. triacantha

13. Hojas de (10-)11,5-14,5(-16) x (7-)7,5-9,5(-10) mm. Segmento foliar central de 4-7,5(-8) mm de largo, relación largo/ancho menor a 7

14(13). Cojines chatos y compactos. Frutos muy comprimidos dorsalmente. Segmentos foliares angostamente ovados a ovados A. triacantha var. famatinense

14. Matas bajas y laxas. Frutos poco comprimidos dorsalmente. Segmentos foliares angostamente triangulares a triangulares A. ulicina

15(1). Plantas androdioicas (algunos individuos presentan umbelas con flores exclusivamente estaminadas, otros, umbelas con flores estaminadas en la periferia y perfectas en el centro)

15. Plantas monoicas o andromonoicas (individuos con umbelas de flores perfectas, o individuos con umbelas de flores exclusivamente estaminadas y umbelas de flores perfectas)

16(15). Hojas rómbicas, divididas en 3(-5) lóbulos triangulares. Umbelas 5-40-floras. Chile A. spinosa 16. Hojas lineales, enteras o divididas en 3 lóbulos aciculares. Umbelas (3-)5-10(-15)-floras. Argentina y Chile A. crypthanta

17(15). Cara abaxial de la lámina brillante. Hojas persistentes, esclerosándose a la madurez y persistiendo largo tiempo sobre las ramas. Umbelas (1-)2(-3)-floras

17. Cara abaxial de la lámina opaca. Pecíolo y/o base foliar persistentes, lámina foliar caduca, aunque persisten sobre las ramas restos de las bases foliares o también de los pecíolos. Umbelas 1-40-floras 20 18(17). Hojas 3-sectas, glabras en ambas caras, base foliar cilíndrica, cerrada, sin lígula. Argentina y Chile ...... A. lycopodioides 18. Hojas 3(7)-partidas, estrigosas en la cara adaxial, glabras en cara abaxial, base foliar formando una vaina cerrada hacia abajo, ligulada en la articulación con la lámina. Argentina, Chile e islas subantárticas 19 19(18). Lámina foliar dividida en (4-)5-6(-7) lóbulos obtusos, que terminan en un pequeño mucrón. Umbelas 3-floras. Frutos de 1,7-2 mm de largo, sobresaliendo de las hojas. Sépalos persistentes A. selago 19. Lámina foliar dividida en 3(-5) lóbulos agudos, que terminan en una seta larga. Umbelas 1(2)-floras. Frutos mayores a 1,7 $\mathrm{mm}$ de largo, escondidos entre las hojas. Sépalos generalmente deciduos A. macquariensis 20(17). Lámina foliar entera

20. Lámina foliar crenada, serrada o débil hasta profundamente incisa .............................................................. 25

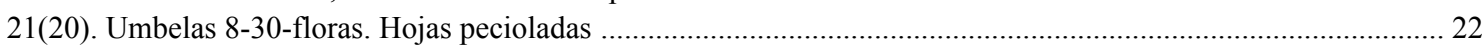

21. Umbelas 1-5-floras. Hojas generalmente sésiles ...................................................................................... 23

22(21). Frutos de 4-6 x 3-3,5 mm. Hojas generalmente glabras. Argentina .............................................. A. patagonica

22. Frutos de 2,5-4 x 2-2,5 mm. Hojas generalmente estrigosas. Costa Rica, Ecuador, Perú, Bolivia y Argentina ......... A. biloba 23(21). Margen de la base foliar y/o de las brácteas con abundantes cilias aracnoides. Especie de regiones templadas. Argentina y Chile A. monantha

23. Margen de la base foliar y/o de las brácteas glabro o con escasas cilias. Especies de regiones tropicales o subtropicales 
24(23). Costillas filiformes (canales secretores del fruto poco desarrollados). Cojines pequeños de hasta $10 \mathrm{~cm}$ de altura. Perú y Bolivia

A. diapensioides

24. Costillas gruesas (canales secretores del fruto muy desarrollados). Cojines extensos de hasta $100 \mathrm{~cm}$ de altura. Perú, Bolivia, Chile y Argentina

A. compacta

25(20). Hojas pequeñas (lámina y pecíolo generalmente menores a $10 \mathrm{~mm}$ de largo), semi-rígidas o rígidas, punzantes (excepto A. madreporica); sésiles, o, si pecioladas, el pecíolo es corto, ancho y la base del limbo, decurrente, se continua insensiblemente con él

25. Hojas mayores (lámina y pecíolo generalmente mayores a $10 \mathrm{~mm}$ de largo), cartáceas o coriáceas, generalmente no punzantes; pecioladas, el pecíolo es corto o largo, delgado, y netamente articulado con la lámina 31 26(25). Pedicelos gruesos (iguales o poco menores en su porción distal que el ancho del fruto). Cojines de hasta $100 \mathrm{~cm}$ de altura 27

26. Pedicelos delgados (menores en su porción distal que el ancho del fruto). Cojines menores de $50 \mathrm{~cm}$ de altura ........... 28 27(26). Lámina foliar 3-partida, glabra o con pocos pelos esparcidos en la cara adaxial. Umbelas 4-7(-14)-floras. Colombia, Ecuador y Perú A. corymbosa

27. Lámina foliar 5-partida, densamente estrigosa en la cara adaxial. Umbelas 1-4-floras. Perú y Bolivia .... A. pulvinata 28(26). Pedicelos de hasta $3 \mathrm{~mm}$ de largo en las umbelas fructíferas. Umbelas sésiles o subsésiles (pedúnculos de hasta $2 \mathrm{~mm}$ de largo), 2-10-floras

28. Pedicelos mayores de $5 \mathrm{~mm}$ de largo en las umbelas fructíferas. Umbelas pedunculadas (pedúnculos de 3-25 $\mathrm{mm}$ de largo), 5-14-floras, o sésiles o subsésiles, 10-15-floras 30

29(28). Base foliar conspicuamente pulvinada. Hojas rígidas y punzantes. Lámina glabra. Dientes del cáliz obsoletos A. crassipes

29. Base foliar no pulvinada o algo pulvinada. Hojas crasas, no punzantes. Lámina generalmente vellosa. Dientes del cáliz evidentes A. madreporica 30(28). Umbelas sésiles o subsésiles (pedúnculos de hasta $2 \mathrm{~mm}$ de largo). Fruto 2-2,5 x 1,7-2 mm. Colombia y Ecuador A. pedunculata 30. Umbelas fructíferas pedunculadas (pedúnculos de 3-25 de largo). Fruto 2,5-3 x 2-2,5 mm. Argentina y Chile A. trifurcata

31(25). Lámina foliar débilmente bilobada en el ápice. Costa Rica, Ecuador, Perú, Bolivia y Argentina A. biloba

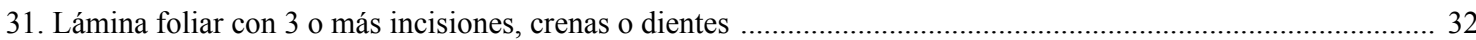

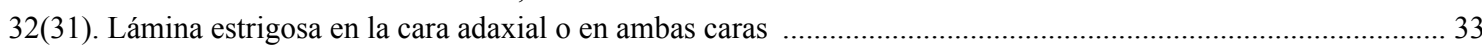

32. Lámina glabra o con algunos pelos restringidos al margen o a la articulación con el pecíolo .............................. 35

33(32). Lámina foliar crenada, crenas ovadas. Venezuela, Colombia, Ecuador y Perú ................................... A. crenata

33. Lámina foliar serrada o inciso-serrada, dientes triangulares ............................................................................. 34

34(33). Umbelas pedunculadas, pedúnculos de 10-60 mm de largo. Lámina foliar con (7)9-21 dientes, no punzantes, prolongados en un pelo conspicuo de hasta $4 \mathrm{~mm}$ de largo. Venezuela y Colombia ...................................cuatrecasasii 34. Umbelas sésiles o subsésiles, pedúnculos de hasta $2 \mathrm{~mm}$ de largo. Lámina foliar con (3)5-7 dientes, punzantes, espinosos. Venezuela, Colombia, Ecuador, Perú y Bolivia A. multifida

35(32). Umbelas 6-10-floras. Andes de Venezuela a Ecuador 36

35. Umbelas 10-40-floras. Andes patagónicos 37

36(35). Lámina foliar profundamente 3(5) lobada. Colombia y Ecuador A. aretioides

36. Lámina foliar crenada. Venezuela y Colombia A. julianii

37(35). Lámina foliar trisecta, lóbulos profundamente divididos en segmentos angostos. Argentina y Chile A. trifoliolata

37. Lámina foliar serrada, dentada, o profundamente dividida en tres lóbulos enteros o dentados 38 38(37). Fruto 2,2-4 x 2-2,5 mm, muy comprimido dorsalmente. Lámina foliar con 3-5 dientes o crenas en el ápice, hasta 3-lobada, lóbulos enteros o dentados, no punzantes. Argentina y Chile 39 38. Fruto 2 x 1,5 mm, poco comprimido dorsalmente. Lámina foliar con 7-11 dientes, punzantes. Chile ............. A. monteroi 39(38). Lámina foliar dentada, dientes de 1,75-3,5 mm de largo A. diversifolia var. diversifolia 39. Lámina profundamente dividida, lóbulos de 4-11 mm de largo A. diversifolia var. antillanca 
Tabla 1. Lista de las especies, variedades y subespecies aceptadas en cada subgénero, incluyendo el nombre tradicionalmente utilizado y aceptado previamente en las referencias aquí citadas, su distribución geográfica, y las referencias a las revisiones taxonómicas o florísticas donde se encuentran descripciones morfológicas e iconografía para cada taxón.

Azorella subgénero Azorella

Nombre actualmente aceptado

Nombre tradicionalmente utilizado

Huanaca acaulis Cav.

Schizeilema allanii Cheeseman Nueva Zelanda

3. Azorella ameghinoi Speg.

4. Azorella andina (Phil.) Drude Azorella boelckei (Mathias \&

5. Constance) G.M. Plunkett \& A.N. Nicolas

Azorella burkartii (Mathias \&

6. Constance) G.M. Plunkett \& A.N. Nicolas

7. Azorella cockaynei Diels

8. Azorella colensoi (Domin) G.M. Plunkett \& A.N. Nicolas

9. Azorella exigua (Hook.f.) Drude

10. Azorella filamentosa Lam.

11. Azorella fuegiana Speg.

12. Azorella fragosea (F. Muell.) Druce

13.a. Azorella haastii (Hook.f.) Drude subssp. haastii

Azorella haastii subssp.

13.b. cyanopetalum (Domin) G.M. Plunkett \& A.N. Nicolas

14.a. Azorella hookeri Drude var. hookeri

Azorella hookeri var. tripartita

14.b. (Hook.f.) G.M. Plunkett \& A.N. Nicolas

15. Azorella hydrocotyloides
(Hook.f.) Kirk

16. Azorella lyallii (Armstr.) G.M. Plunkett \& A.N. Nicolas

17. Azorella nitens Petrie

18. Azorella pallida (Kirk) Kirk

Azorella polaris (Hombr. \&

19. Jacq.) G.M. Plunkett \& A.N. Nicolas
Azorella ameghinoi Speg.

Huanaca andina (Phil.) Phil.

Huanca boelckei Mathias \& Constance

Huanaca burkartii Mathias \& Constance

Schizeilema cockaynei (Diels) Cheeseman

Schizeilema colensoi Domin

Schizeilema exiguum (Hook.f.) Domin

Azorella filamentosa Lam.

Azorella fuegiana Speg.

Schizeilema fragoseum (F. Muell.) Domin

Schizeilema haastii (Hook.f.)

Domin subssp. hookerianum

Domin.

Schizeilema haastii (Hook.f.)

Domin subssp. cyanopetalum

Domin

Schizeilema trifoliolatum (Hook.f.) Domin

Schizeilema trifoliolatum

(Hook.f.) Domin

Schizeilema hydrocotyleoides (Hook.f.) Domin

Stilbocarpa lyallii Armstr.

Schizeilema nitens (Petrie)

Domin

Schizeilema pallidum (Kirk)

Domin Stilbocarpa polaris (Hombr. \&
Jacq.) A. Gray
Distribución geográfica

Argentina y Chile

Argentina y Chile

Martínez (1989)

Revisión taxonómica o

florística

Mathias \& Constance (1971)

Allan (1961)

Mathias \& Constance (1971)

Argentina y Chile

Mathias \& Constance (1971)

Mathias \& Constance (1971)

Nueva Zelanda

incluyendo Islas Stewart

Allan (1961)

Nueva Zelanda

Allan (1961)

Nueva Zelanda

Allan (1961)

Argentina incluyendo Islas Malvinas y Chile

Martínez (1989)

Argentina y Chile

Martínez (1989)

Australia

Jacobs \& Pickard (1981);

PlantNET (2019)

Nueva Zelanda incluyendo Islas Stewart

Allan (1961)

Nueva Zelanda incluyendo Islas Stewart

Allan (1961)

Nueva Zelanda incluyendo Islas Stewart

Allan (1961)

Nueva Zelanda incluyendo

Islas Stewart

Allan (1961)

Nueva Zelanda

Allan (1961)

Nueva Zelanda incluyendo Islas Stewart

Allan (1961)

Nueva Zelanda

Allan (1961)

Nueva Zelanda

Allan (1961)

Islas Subantárticas

de Australia y Nueva

Allan (1961), Zelanda (Islas Antípodas, Mitchell et al. (1999)
Argentina y Chile 
Tabla 1. (Continuación). Lista de las especies, variedades y subespecies aceptadas en cada subgénero, incluyendo el nombre tradicionalmente utilizado y aceptado previamente en las referencias aquí citadas, su distribución geográfica, y las referencias a las revisiones taxonómicas o florísticas donde se encuentran descripciones morfológicas e iconografía para cada taxón.
20. Azorella ranunculus d'Urv
21. Azorella robusta (Kirk) G.M.
21. Plunkett \& A.N. Nicolas
Schizeilema ranunculus (d'Urv.) Domin
Argentina incluyendo Islas Malvinas y Chile
Constance (1988)
Stilbocarpa robusta (Kirk)
Cockayne
Nueva Zelanda
Allan (1961),
(Islas Snares)
Mitchell et al. (1999)
22. Azorella roughii (Hook.f.) Kirk
Schizeilema roughii (Hook.f.)
Domin
Nueva Zelanda
Allan (1961)
23. Azorella schizeilema G.M.
Schizeilema reniforme
(Hook.f.) Domin
Nueva Zelanda (Islas
Auckland y Campbell)
Allan (1961)

\section{Azorella subgénero Andinae}

Nombre actualmente aceptado

*Azorella albovaginata (Gillies

24. \& Hook.) G.M. Plunkett \& A.N. Nicolas

25. Azorella aretioides DC.

26. Azorella biloba (Schltdl.) Wedd.

27. Azorella compacta Phil.

28. Azorella corymbosa (Ruiz \& Pav.) Pers.

29. Azorella crassipes Phil.

30. Azorella crenata (Ruiz \& Pav.) Pers.

31. Azorella cryptantha (Clos) Reiche

32. Azorella cuatrecasasii Mathias \& Constance

33. Azorella diapensioides A. Gray

34.a. Azorella diversifolia Clos var.

diversifolia

34.b. *Azorella diversifolia var. antillanca J.C. Zech

35. Azorella echegarayi (Hieron.)

35. G.M. Plunkett \& A.N. Nicolas 36. Azorella hallei (Skottsb.) G.M.
Plunkett \& A.N. Nicolas 37. Azorella julianii Mathias \&
Constance

38. Azorella lycopodioides Gaudich.

39. Azorella macquariensis Orchard
Nombre tradicionalmente utilizado

Mulinum albovaginatum Gillies \& Hook.

Azorella aretioides DC.

Azorella biloba (Schltdl.) Wedd.

Azorella compacta Phil.

Azorella corymbosa (Ruiz \& Pav.) Pers.

Azorella crassipes Phil.

Azorella crenata (Ruiz \& Pav.) Pers.

Azorella cryptantha (Clos)

Reiche

Azorella cuatrecasasii Mathias \& Constance

Azorella diapensioides A. Gray Perú y Bolivia

Argentina y Chile

Chile

Revisión taxonómica o

Distribución geográfica florística

Argentina y Chile

Fernández et al. (2017c)

Colombia, Ecuador (Panamá, Perú, Costa Rica?)

Calviño et al. (2016)

Costa Rica, Ecuador, Perú, Bolivia y Argentina

Martínez (1989)

Perú, Bolivia, Chile y Argentina

Martínez (1989)

Colombia, Ecuador y Perú Calviño et al. (2016)

Argentina y Chile

Martínez (1989)

Venezuela, Colombia,

Ecuador y Perú

Calviño et al. (2016)

Argentina y Chile

Martínez (1989)

Azorella diversifolia Clos var. diversifolia

Azorella diversifolia var. antillanca J.C. Zech

Mulinum echegarayi Hieron. Argentina

Argentina

Mulinum hallei Skottsb.

Azorella julianii Mathias \& Constance

Azorella lycopodioides

Gaudich.

Azorella macquariensis Orchard

\section{Venezuela y Colombia}

Calviño et al. (2016)

Argentina incluyendo Islas Malvinas y Chile

Martínez (1989)

Australia (Isla Macquarie) Orchard (1989)

* Diferencias nomenclaturales y/o taxonómicas respecto del trabajo de Plunkett \& Nicolas [2017 (2016 on-line)]. 
Tabla 1. (Continuación). Lista de las especies, variedades y subespecies aceptadas en cada subgénero, incluyendo el nombre tradicionalmente utilizado y aceptado previamente en las referencias aquí citadas, su distribución geográfica, y las referencias a las revisiones taxonómicas o florísticas donde se encuentran descripciones morfológicas e iconografía para cada taxón.
40. Azorella madreporica $\mathrm{Clos}$
Azorella madreporica Clos
Argentina y Chile
Martínez (1989)
41. Azorella microphylla (Cav.)
Mulinum microphyllum (Cav.)
Pers.
Argentina
Fernández et al. (2017c)
42. Azorella monantha Clos
Azorella monantha Clos
Argentina incluyendo Islas
Malvinas y Chile
Martínez (1989)
43. Azorella monteroi S. Martínez
Azorella monteroi S. Martínez
\& Constance
Chile
Calviño et al. (2016)
44. Azorella multifida (Ruiz \& Pav.) Azorella multifida (Ruiz \&
Venezuela, Colombia,
Ecuador, Perú y Bolivia
Calviño et al. (2016)
45. Azorella nivalis Phil.
Mulinum leptacanthum Phil.
Argentina y Chile
Fernández et al. (2017c)
46. Azorella patagonica Speg.
Azorella patagonica Speg.
Argentina
Martínez (1989)
47. Azorella pedunculata (Spreng.)
Azorella pedunculata (Spreng.) Mathias \& Constance
Colombia y Ecuador
Calviño et al. (2016)
*Azorella prolifera (Cav.) G.M.
48.a. Plunkett \& A.N. Nicolas var. prolifera
Mulinum spinosum (Cav.) Pers. var spinosum
Argentina y Chile
Fernández et al. (2017c)
* Azorella prolifera var. hirsuta 48.b. (Phil.) Mar. Fernández \& C.I.
Calviño
Mulinum spinosum var
hirsutum Mar. Fernández \& Chile
C.I. Calviño
Fernández et al. (2017c)
*Azorella prolifera var. zechii
48.c. (Mar. Fernández \& C.I. Calviño)
Mar. Fernández \& C.I. Calviño
Mulinum spinosum var zechii
Mar. Fernández \& C.I. Calviño Chile
Fernández et al. (2017c)
49. Azorella pulvinata Wedd.
Azorella pulvinata Wedd.
Perú y Bolivia
Calviño et al. (2016)
50. Azorella ruizii G.M. Plunkett \&
Laretia acaulis (Cav.) Gillies
\& Hook.
Argentina y Chile
Fernández et al. (2016b)
Argentina incluyendo Islas
51. Azorella selago Hook.f.
Azorella selago Hook.f.
Malvinas, Chile e Islas
Martínez (1989)
Subantárticas
52. Azorella spinosa (Ruiz \& Pav.)
Pers.
Azorella spinosa (Ruiz \& Pav.)
Pers.
Chile
Calviño et al. (2016)
*Azorella triacantha (Griseb.)
53.a. Mar. Fernández \& C.I. Calviño var. triacantha
Mulinum triacanthum Griseb.
var triacanthum
Bolivia y Argentina
Fernández et al. (2017c)
* Azorella triacantha var.
53.b. famatinense (H. Wolff) Mar. Fernández \& C.I. Calviño
Mulinum triacanthum var
famatinense (H. Wolff) Mar.
Fernández \& C.I. Calviño
Argentina
Fernández et al. (2017c)
54. Azorella trifoliolata Clos
Azorella trifoliolata Clos
Argentina y Chile
Martínez (1989)
55. Azorella trifurcata (Gaertn.)
Azorella trifurcata (Gaertn.)
Pers.
Argentina y Chile
Martínez (1989)
56. *Azorella trisecta (H. Wolff) Mar. Fernández \& C.I. Calviño
Mulinum crassifolium Phil.
Argentina y Chile
Fernández et al. (2017c)
*Azorella ulicina (Gillies \&
57. Hook.) G.M. Plunkett \& A.N. Nicolas
Mulinum ulicinum Gillies \&
Hook.
Argentina y Chile
Fernández et al. (2017c)
Mulinum valentini Speg.
Argentina y Chile
Fernández et al. (2017c)
58. *Azorella valentini (Speg.) Mar. Fernández \& C.I. Calviño

* Diferencias nomenclaturales y/o taxonómicas respecto del trabajo de Plunkett \& Nicolas [2017 (2016 on-line)]. 
Lista de especies aceptadas en Azorella subgen. Andinae con sus sinónimos.

Azorella albovaginata (Gillies \& Hook.) G.M. Plunkett \& A.N. Nicolas, Brittonia 69: 48. 2017 (2016 on-line). Mulinum albovaginatum Gillies \& Hook., Bot. Misc. 1: 328. 1830.

Mulinum pauciflorum Reiche, Anales Univ. Chile 104: 803. 1899. Mulinum reichei H. Wolff, Repert. Spec. Nov. Regni Veg. 17: 441. 1921, nom. illeg. superfl. Mulinum albovaginatum var. pauciflorum (Reiche) Zech, Kurtziana 24: 192. 1995. Azorella albovaginata var. pauciflorum (Reiche) G.M. Plunkett \& A.N. Nicolas, Brittonia 69: 48. 2017 (2016 on-line).

Mulinum cryptanthum Clos var. pulvinaris Chodat \& Wilcz., Bull. Herb. Boissier sér. 2, 2: 525.1902.

\section{Azorella aretioides DC., Prodr. 4: 77. 1830.}

Azorella aretioides var. elongata Wedd., Chlor. Andina 2: 195. 1860.

Observaciones. Plunkett \& Nicolas [2017 (2016 on-line)] amplían la distribución de esta especie a Panamá, Perú y Costa Rica, pero no citan material examinado. Dado que esta especie puede ser confundida con Azorella biloba, cuya distribución es más amplia, y hasta tanto no podamos confirmar las determinaciones, consideramos esta distribución dudosa (Tabla 1).

Azorella biloba (Schltdl.) Wedd., Chlor. Andina 2: 195. 1860. Fragrosa biloba Schltdl., Linnaea 28: 478. 1856[1857].

Azorella biloba var. strigosa Wedd., Chlor. Andina 2: 196.1860.

Azorella lehmannii Hieron., Bot. Jahrb. Syst. 20: 71. 1895.

Azorella compacta Phil., Anales Mus. Nac. Santiago de Chile 8: 28. 1891. Laretia compacta (Phil.) Reiche. Anales Univ. Chile 104: 784. 1899.
Azorella prismatoclada Domin. Repert. Spec. Nov. Regni Veg. 4: 297. 1907.

Azorella columnaris H. Wolff, Bot. Jahrb. Syst. 40: 288. 1908.

Azorella yareta Hauman, Physis (Buenos Aires) 4: 485. 1919. Laretia yareta (Hauman) Mathias \& Constance, Univ. Calif. Publ. Bot. 29: 215. 1957.

Azorella corymbosa (Ruiz \& Pav.) Pers., Syn. P1. 1: 305. 1805. Fragosa corymbosa Ruiz \& Pav., Fl. Peruv. 3: 27, tab. 250, fig. a. 1802.

Azorella crassipes Phil., Anales Univ. Chile 85: 702. 1894.

Azorella crenata (Ruiz \& Pav.) Pers., Syn. Pl. 1: 302. 1805. Fragosa crenata Ruiz \& Pav., Fl. Peruv. 3: 27, tab. 249, fig. c. 1802. Bolax crenatus (Ruiz \& Pav.) Spreng., en Roem. \& Schult., Syst. Veg. 6: 362. 1820.

Fragosa cladorrhiza Ruiz \& Pav., Fl. Peruv. 3: 27, tab. 250, fig. b. 1802. Azorella cladorrhiza (Ruiz \& Pav.) Pers., Syn. Pl. 1: 303. 1805. Bolax cladorrhizus (Ruiz \& Pav.) Spreng., Syst. Veg., ed. 15 bis 6: 362.1820 .

Fragosa reniformis Ruiz \& Pav., Fl. Peruv. 3: 26, tab. 249, fig. b. 1802. Azorella reniformis (Ruiz \& Pav.) Pers., Syn. Pl. 1: 303. 1805.

Azorella crenata var. compacta Wedd., Chlor. Andina 2: 195. 1860.

Azorella crenata var. mutisiana Hieron., Bot. Jahrb. Syst. 20: Beibl. 49: 71. 1895. Azorella mutisiana (Hieron.) Hieron. ex H. Wolff, Repert. Spec. Nov. Regni Veg. 17: 266. 1921.

Azorella ecuadorensis Domin, Repert. Spec. Nov. Regni Veg. 4: 296. 1907.

Azorella vareschii Suess. \& Launert, Mitt. Bot. Staatssamml. München 2: 82, fig. 2. 1955.

Observaciones. Mantenemos a Azorella reniformis como sinónimo de $A$. crenata, como se detalla en Calviño et al. (2016). Sin embargo, este taxón está siendo estudiado por las autoras y S. Beck ya que podría tratarse de una especie válida de Azorella. 
Azorella cryptantha (Clos) Reiche, Bot. Jahrb. Syst. 28(1): 17. 1899. Mulinum cryptanthum Clos, Fl. Chil. 3(1): 90. 1847[1848].

Mulinum integrifolium Hieron., Bol. Acad. Nac. Ci. Córdoba 4: 28. 1881.

Azorella cuatrecasasii Mathias \& Constance, Bull. Torrey Bot. Club 89(6): 371, fig. 1. 1962.

Azorella diapensioides A. Gray, Bot. Wilkes Exped. 1: 702. 1854.

Azorella diapensioides var. denticulata Wedd., Chlor. Andina 2: 191. 1860.

Azorella diapensioides var. glabra Wedd., Chlor. Andina 2: 190. 1860.

Azorella diversifolia Clos, Fl. Chil. 3(1): 86. $1847[1848]$.

\section{Azorella diversifolia var. diversifolia}

Pozoa incisa Griseb., Abh. Königl. Ges. Wiss. Göttingen 6: 123. 1856. Azorella incisa (Griseb.) Wedd. Chlor. Andina 2: 197. 1860.

Azorella diversifolia var. antillanca J. C. Zech, Madroño 44(2): 193. 1997.

Azorella echegarayi (Hieron.) G.M. Plunkett \& A.N. Nicolas, Brittonia 69: 49. 2017 (2016 on-line). Mulinum echegarayi Hieron., Bol. Acad. Nac. Ci. Córdoba 4: 27. 1881.

Azorella hallei (Skottsb.) G.M. Plunkett \& A.N. Nicolas, Brittonia 69: 51. 2017 (2016 on-line). Mulinum hallei Skottsb., Kongl. Svenska Vetensk. Acad. Handl. 56(5): 278. 1916.

Azorella julianii Mathias \& Constance, Fieldiana Bot. 28(2): 445. 1952.
Azorella lycopodioides Gaudich., Ann. Sci. Nat. (Paris) 5: 105, tab. 3. fig. 1. 1825.

Azorella lycopodioides var. chilensis Clos, Fl. Chil. 3(1): 83. 1847[1848].

Azorella nervosa Phil., Linnaea 28: 696. 1856.

Azorella clandestina Phil., Anales Univ. Chile 85: 701.1894.

Azorella laevigata Phil., Anales Univ. Chile 85: 700. 1894.

Azorella lycopodioides var. compacta Phil., Anales Univ. Chile 85: 702. 1894.

Azorella vaginata Phil., Anales Univ. Chile 85: 704. 1894. Azorella lycopodioides var. vaginata (Phil) Reiche, Anales Univ. Chile 104: 792. 1899.

Azorella philippi Gand., Bull. Soc. France 59: 709. 1913.

Azorella macquariensis Orchard, Muelleria 7(1): 16.1989.

Azorella madreporica Clos, Fl. Chil. 3(1): 79. 1847[1848].

Azorella bolacina Clos, Fl. Chil. 3(1): 80. 1847[1848]. Azorella muscoides Phil., Linnaea 28: 695. 1856. Azorella madreporica var. muscoides (Phil.) Reiche, Anales Univ. Chile 104: 792. 1899. Azorella piligera Phil., Linnaea 28: 652. 1856. Azorella obtusiloba Phil., Anales Univ. Chile 85: 701. 1894.

Azorella microphylla (Cav.) G.M. Plunkett \& A.N. Nicolas, Brittonia 69: 51. 2017 (2016 on-line). Selinum microphyllum Cav., Icon. 5: 59, tab. 486, fig. 2. 1799, nom. \& typ. cons (Fernández et al., 2016). Mulinum microphyllum (Cav.) Pers., Syn. Pl. 1: 309. 1805.

Huanaca morenonis Kuntze, Revis. Gen. Pl. 3(3): 113. 1898. Diplaspis morenonis (Kuntze) K. Schum., Bot. Jahresber. (Just). 26(1): 367. 1900. Mulinum morenonis (Kuntze) Speg., Anales Mus. Nac. Buenos Aires 7: 295. 1902. Azorella morenonis (Kuntze) Macloskie, Rep. Princeton Univ. Exped. Patagonia, Botany 8: 629. 1905. 
Azorella monantha Clos, Fl. Chil. 3(1): 79. 1847[1848].

Azorella hookeriana Clos, Fl. Chil.3(1): 81.1847[1848]. Azorella apoda A. Gray, U.S. Expl. Exped., Phan. 15: 699. 1854.

Azorella bryoides Phil., Linnaea 28: 695. 1856. Azorella sessiliflora Phil., Linnaea 28: 652. 1856. Apleura nucamentacea Phil., Linnaea 33: 90. 1864. Azorella nucamentacea (Phil.) Hauman, Physis (Buenos Aires) 4: 483. 1919.

Azorella glacialis Phil., Anales Univ. Chile 85: 704. 1894.

Azorella monteroi S. Martínez \& Constance, Gayana, Bot. 48: 117. 1991.

Azorella multifida (Ruiz \& Pav.) Pers., Syn. Pl. 1: 303. 1805. Fragosa multifida Ruiz \& Pav. Fl. Peruv. 3: 27, pl. 249. fig. 2. 1802. Bolax multifidus (Ruiz \& Pav.) Spreng., en Roem. \& Schult. Syst. Veg. 6: 362. 1820.

Azorella laxa H. Wolff, Bot. Jahrb. Syst. 40: 288. 1908. Azorella weberbaueri H. Wolff, Bot. Jahrb. Syst. 40: 287.1908.

Azorella nivalis Phil., Anales Univ. Chile 85: 703-704. 1894.

Mulinum leptacanthum Phil., Anales Univ. Chile 85: 709. 1894.

Mulinum patagonicum Speg., Anales Soc. Ci. Argent. 48: 56-57. 1899.

Azorella patagonica Speg., Anales Mus. Nac. Buenos Aires 4: 293. 1902.

Azorella patagonica var. compacta Speg., Anales Mus. Nac. Buenos Aires 4: 294. 1902.

Azorella pedunculata (Spreng.) Mathias \& Constance, Mem. New York. Bot. Gard. 9: 172. 1955. Bolax pedunculatus Spreng., Sp. Umbell.: 10. 1818.
Bolax pilifera Turcz., Bull. Soc. Imp. Naturalistes Moscou 22(2): 32. 1849.

Azorella prolifera (Cav.) G.M. Plunkett \& A.N. Nicolas, Brittonia 69: 49. 2017 (2016 on-line). Selinum proliferum Cav., Icon. 5: 59, tab. 486, fig. 1. 1799. Mulinum proliferum (Cav.) Pers., Syn. Pl. 1: 309. 1805. Bolax prolifer (Cav.) Spreng., Syst. Veg. 6: 361. 1820. Mulinum spinosum var. proliferum (Cav.) Kuntze, Revis. Gen. Pl. 3(3): 114. 1898.

\section{Azorella prolifera var. prolifera}

Selinum spinosum Cav., Icon. 5: 59, tab. 487, f. 1. 1799. Mulinum spinosum (Cav.) Pers. Syn. Pl. 1: 309. 1805. Bolax spinosus (Cav.) Spreng. Syst. Veg. 6: 362. 1820.

Mulinum echinus DC., Prodr. 4: 79. 1830. Azorella echinus (DC.) G.M. Plunkett \& A.N. Nicolas, Brittonia 69: 19. 2017 (2016 on-line).

Mulinum laxum Phil., Linnaea 33: 90. 1864. Mulinum spinosum var. laxum (Phil.) Reiche, Anales Univ. Chile 104: 802. 1899.

Mulinum leoninum Lorentz, in Roca, Exped. Río Negro, Bot. 222, lám. 6(1), figs. 1-5. 1883. Mulinum spinosum var. leoninum (Lorentz) Kuntze, Revis. Gen. Pl. 3(3): 114. 1898.

Mulinum chillanense Phil., Anales Univ. Chile 85: 707. 1894. Mulinum spinosum var. chillanense (Phil.) Reiche, Anales Univ. Chile 104: 802. 1899.

Mulinum ovalleanum Phil., Anales Univ. Chile 85: 709. 1894.

Mulinum spinosum var. leoninum (Lorentz) Kuntze f. toscae Kuntze, Revis. Gen. Pl. 3(3): 114. 1898.

Mulinum spinosum var. quinquepartitum Kuntze, Revis. Gen. Pl. 3 (3): 114. 1898.

Mulinum spinosum var. triacanthum (Griseb.) Kuntze f. minus Kuntze, Revis. Gen. Pl. 3(3): 114. 1898. Mulinum spinosum var. minus (Kuntze) Zech, Kurtziana 24: 192. 1995.

Mulinum spinosum var. trispinescens Kuntze, Revis. Gen. Pl. 3(3): 114. 1898.

Mulinum patagonicum Gand., Bull. Soc. Bot. France 59: 710. 1913, nom. illeg. hom. Mulinum gandogeri Hiroe, Umbell. World 1732. 1979.

Mulinum spinosum var. longeinvolucratum Hicken, Darwiniana 1(2): 61. 1923. 
Azorella prolifera var. hirsuta (Phil.) Mar. Fernández \& C.I. Calviño. comb. nov. Basónimo: Mulinum hirsutum Phil. Anales Univ. Chile 85: 708. 1894. Mulinum spinosum var. hirsutum (Phil.) Mar. Fernández \& C.I. Calviño, Anales Jard. Bot. Madrid 74(1): 21. 2017.

Observaciones. Plunkett \& Nicolas [2017 (2016 on-line)] consideran el nombre Mulinum hirsutum Phil. un homónimo ilegítimo de Mulinum hirsutum Meyen ex Walp. (1843). Este último es un nomen nudum, sin publicación válida, por lo que el nombre de Philippi es ilegítimo.

Azorella prolifera var. zechii (Mar. Fernández \& C.I. Calviño) Mar. Fernández \& C.I. Calviño comb. nov. Basónimo: Mulinum spinosum var. zechii Mar. Fernández \& C.I. Calviño, Anales Jard. Bot. Madrid 74(1): 21. 2017.

Azorella pulvinata Wedd., Chlor. Andina 2: 194, lam. 66A. 1860.

Azorella ruizii G.M. Plunkett \& A.N. Nicolas, Brittonia 69: 52.2017 (2016 on-line). Selinum acaule Cav., Icon. 5: 59-60, tab. 487, f. 2. 1799. Mulinum acaule (Cav.) Pers., Syn. Pl. 1: 309. 1805. Laretia acaulis (Cav.) Gillies \& Hook., Bot. Misc. 1: 329, tab. 65. 1830.

Azorella selago Hook. Fl. Antarct. 2(2): 284, pl. 99. 1844.

Azorella selago var. compacta Alboff, Rev. Mus.

La Plata 7: 371. 1896.

Azorella selago var. pulvinaris Alboff, Rev. Mus.

La Plata 7: 371. 1896.

Azorella antipoda Gand., Bull. Soc. Bot. France

72: 178. 1925.

Azorella spinosa (Ruiz \& Pav.) Pers., Syn. Pl. 1: 303. 1805. Fragosa spinosa Ruiz \& Pav., Fl. Peruv. 3: 27. 1802.
Mulinum cuneatum Hook. \& Arn., Bot. Beechey Voy. 26. 1830.

Azorella pectinata Phil., Anales Univ. Chile 85: 700. 1894.

Mulinum clandestinum Phil., Anales Univ. Chile 85: 708. 1894.

Azorella triacantha (Griseb.) Mar. Fernández \& C.I. Calviño, comb. nov. Basónimo: Mulinum triacanthum Griseb., Abh. Königl. Ges. Wiss. Göttingen 19: 154. 1874. Mulinum spinosum var. triacanthum (Griseb.) Kuntze. Revis. Gen. Pl. 3(3): 114. 1898.

\section{Azorella triacantha var. triacantha.}

Mulinum axilliflorum Griseb., Abh. Königl. Ges. Wiss. Göttingen 19: 154. 1874.

Azorella triacantha var. famatinense $(\mathrm{H}$. Wolff) Mar. Fernández \& C.I. Calviño, comb. nov. Basónimo: Mulinum famatinense H. Wolff, Repert. Spec. Nov. Regni Veg. 17: 441. 1921. Mulinum triacanthum var. famatinense $(\mathrm{H}$. Wolff) Mar. Fernández \& C.I. Calviño, Anales Jard. Bot. Madrid, 74(1): 23. 2017.

Azorella trifoliolata Clos, Fl. Chil. 3(1): 85, t. 30. fig. 2. 1847[1848].

Azorella gayana Phil., Linnaea 28: 653. 1856. Azorella depauperata Phil., Fl. Atacam.: 24. 1860. Huanaca lechleri Kuntze, Revis. Gen. Pl. 3(3): 113. 1898.

Azorella pinnatiloba Gand., Bull. Soc. Bot. France 59: 710. 1913.

Azorella trifurcata (Gaertn.) Pers., Syn. Pl. 1: 303. 1805. Chamitis trifurcata Gaertn., Fruct. Sem. Pl. 1: 95. 1788. Bolax trifurcatus (Gaertn.) Spreng. ex Steud., Nomencl. Bot. 1: 214. 1840.

Azorella cespitosa Cav., Icon. 5: 57, tab. 484, fig. 2. 1799, nom. illeg. hom. Azorella crassifolia Pers. Syn. Pl. 1: 303. 1805. Bolax caespitosus Spreng. Syst. Veg. 6: 358. 1820. 
Bolax gilliesii Hook., Bot. Misc. 1: 325, lam. 63. 1830. Azorella gilliesii (Hook.) Hook. \& Arn., Bot. Misc. 3: 347. 1833.

Azorella utriculata Griseb., Abh. Königl. Ges.

Wiss. Göttingen 6: 122. 1854.

Azorella albida Phil., Anales Univ. Chile 85: 706. 1894. Azorella rahmeri Phil., Anales Univ. Chile 85: 703. 1894.

Azorella trisecta (H. Wolff) Mar. Fernández \& C.I. Calviño, comb. nov. Basónimo: Azorellopsis trisecta H. Wolff, Repert. Spec. Nov. Regni Veg. 19: 312. 1924.

Mulinum crassifolium Phil., Fl. Atacam. 24. 1860. Azorella atacamensis G.M. Plunkett \& A.N. Nicolas, Brittonia 69: 48. 2017 (2016 on-line).

Observaciones. Plunkett \& Nicolas [2017 (2016 on-line)] al transferir Mulinum crassifolium Phil. a Azorella, ante la existencia de $A$. crassifolia Pers. (= Azorella monantha Clos), y por no haber otro epíteto disponible, proponen como nombre de reemplazo a $A$. atacamensis. En nuestra opinión el nombre Azorellopsis trisecta $\mathrm{H}$. Wolff se aplica a este taxón, por lo que el epiteto trisecta está disponible y tiene prioridad para establecer la nueva combinación. Consideraciones sobre sinonimia y tipificación de Azorellopsis trisecta se encuentran en Fernández et al. (2017c).

Azorella ulicina (Gillies \& Hook.) G.M. Plunkett \& A.N. Nicolas, Brittonia 69: 50. 2017 (2016 on-line). Mulinum ulicinum Gillies \& Hook., Bot. Misc. 1: 328, t. 64. 1830.

Mulinum triacanthum var. multiflorum Hieron., Bol. Acad. Nac. Ci. Córdoba 4: 27. 1881. Mulinum ulicinum var. multiflorum (Hieron.) Zech, Kurtziana 24: 192. 1995.

Azorella valentini (Speg.) Mar. Fernández \& C.I. Calviño, comb. nov. Basónimo: Mulinum valentini Speg., Anales Soc. Ci. Argent. 48: 57. 1899.

Mulinum lycopodiopsis Speg., Anales Soc. Ci. Argent. 48: 55. 1899.

Azorella concolor Rendle, J. Bot. 42: 368. 1904.
Observaciones. Plunkett \& Nicolas [2017 (2016 on-line)] aplican a esta especie el nombre $A$. concolor Rendle (1904) pero esto no es correcto por cuanto existen dos nombres fundados por Spegazzini (1899) bajo Mulinum, con prioridad de fecha y epítetos finales disponibles, para referirse a la misma: $M$. lycopodiopsis y $M$. valentini. Dado que Constance (1988) fue el primer autor en sinonimizar Mulinum valentini y M. lycopodiopsis y en adoptar el nombre $M$. valentini para el taxon unificado, este último tiene prioridad sobre $M$. lycopodiopsis al momento de adoptar el epíteto para la nueva combinación requerida (ICN Arts. 11.4 y 11.5; Turland et al., 2018).

\section{Nombres dudosos}

Azorella plantaginea Speg., Anales Mus. Nac. Buenos Aires 4: 292. 1902. Este nombre ha sido considerado sinónimo de Azorella patagonica (Constance, 1988; Martínez, 1989, 1993, 2008; Plunkett \& Nicolas, 2017 (2016 on-line)). A partir del análisis de la descripción del protólogo, el tamaño y la consistencia de hojas, en nuestra opinión, este nombre no se corresponde con $A$. patagonica, sino posiblemente, con $A$. diversifolia. Dado que no se localizó material original del nombre que permitiría asignar la sinonimia correcta, se mantiene como un taxón dudoso.

Sobre la base de la revisión taxonómica de Mulinum y Azorella (Calviño et al., 2016; Fernández et al., 2017c), se mantienen los siguientes nombres como dudosos:

Fragosa glabra Lag., Amen. Nat. 2: 93. 1821.

Mulinum spinosum f. humilis Kurtz, Verh. Bot. Vereins Prov. Brandenburg 35: 111. 1894.

Mulinum manifolium Ravenna, Onira 11(21): 56. 2008.

\section{AGRADECIMIENTOS}

Agradecemos a Susana Martínez por su apoyo y sus valiosos comentarios al manuscrito, y a dos revisores anónimos. Este trabajo fue financiado por ANPCyT-FONCyT PICT 2014-0584, CONICET PIP 112-201301-00357, y por la Universidad Nacional del Comahue PIN B205. 


\section{BIBLIOGRAFÍA}

Allan, H. H. 1961. Schizeilema, en H. H. Allan (ed.), Flora of New Zealand, vol. I. Wellington: R.E. Owen Government Printer.

Andersson, L., M. Kocsis \& R. Eriksson. 2006. Relationships of the genus Azorella (Apiaceae) and other hydrocotyloids inferred from sequence variation in three plastid markers. Taxon 55: 270-280.

Cabrera, A. L. 1971. Fitogeografía de la República Argentina. Boletín de la Sociedad Argentina de Botánica 14: 1-50.

Cabrera, A. L. \& A. Willink. 1980. Biogeografía de América Latina. Washington DC: Secretaria General de la Organización de los Estados Americanos.

Calviño, C. I., M. Fernández \& S. G. Martínez. 2016. Las especies de Azorella (Azorelloideae, Apiaceae) con distribución extra-Argentina. Darwiniana 4(1): 57-82.

Constance, L. 1988. Umbelliferae, en M. N. Correa (ed.), Flora Patagónica, Colección Científica del Instituto de Tecnología Agropecuaria 8(5): 310-379.

Fernández, M., S. Martínez \& C. I. Calviño. Typification of names of Azorella (Azorelloideae, Apiaceae) for the Flora of Argentina. Phytotaxa. En prensa.

Fernández, M., S. Martínez \& C. I. Calviño. 2016a. Proposal to conserve the name Selinum microphyllum (Mulinum microphyllum) (Apiaceae: Azorelloideae) with a conserved type. Taxon 65: 396-397.

Fernández, M., C. Ezcurra \& C. I. Calviño. 2016b. Morphology, fruit anatomy and taxonomy of the South Andean genus Laretia (Azorelloideae, Apiaceae). Systematic Botany 41(3): 807-812.

Fernández, M., C. Ezcurra \& C. I. Calviño. 2017a. Chloroplast and ITS phylogenies to understand the evolutionary history of the southern South American Azorella, Laretia and Mulinum (Azorelloideae, Apiaceae). Molecular Phylogenetics and Evolution 108: 1-21.

Fernández, M., C. Ezcurra \& C. I. Calviño. 2017b. Species limits and morphometric and environmental variation within the South Andean and Patagonian Mulinum spinosum species-group (Apiaceae-Azorelloideae). Systematics and Biodiversity 15: 489-505.

Fernández, M., C. Ezcurra \& C. I. Calviño. 2017c. Revisión taxonómica del género sudamericano Mulinum (Azorelloideae, Apiaceae). Anales del Jardín Botánico de Madrid 74(1): 1-36.

Jacobs, S. W. L. \& J. Pickard. 1981. Plants of New South Wales- A census of the Cycads, Conifers and Angiosperms. 226 p. Sydney: National Herbarium of New South Wales, Royal Botanic Gardens.

Mathias, M. \& L. Constance. 1971. A first revision of Huanaca (Umbelliferae-Hydrocotyloideae). Kurtziana 6: 7-23.

Martínez, S. G. 1989. El género Azorella (Apiaceae, Hydrocotyloideae) en la Argentina. Darwiniana 29: 139-178.
Martínez, S. G. 1993. Sinopsis del género Azorella (Apiaceae, Hydrocotyloideae). Darwiniana 32: 171-184.

Martínez, S. G. 1995. Caracteres foliares xeromorfos en Azorella (Apiaceae). Boletín de la Sociedad Argentina de Botánica 30: 167-181.

Martínez, S. 2008. Apiaceae, en F. O. Zuloaga, O. Morrone \& M. J. Belgrano (eds.), Catálogo de las Plantas Vasculares del Cono Sur (Argentina, sur de Brasil, Chile, Paraguay y Uruguay). Monographs in Systematic Botany from the Missouri Botanical Garden107: 1056-1090.

Nicolas, A. N. \& G. M. Plunkett. 2009. The demise of subfamily Hydrocotyloideae (Apiaceae) and the re-alignment of its genera across the entire order Apiales. Molecular Phylogenetics and Evolution 53: 134-151.

Nicolas, A. N. \& G. M. Plunkett. 2012. Untangling generic limits in Azorella, Laretia, and Mulinum (Apiaceae: Azorelloideae): insights from phylogenetics and biogeography. Taxon 61: 826-840.

Philippi, R. A. 1894. Umbelliferae, en R. A. Philippi (ed.), Plantas nuevas chilenas de las familias que corresponden al tomo III de la obra de Gay. Anales de la Universidad de Chile, Memorias Cientificas y Literarias 85: 707-710.

PlantNET (The NSW Plant Information Network System) 2019. Royal Botanic Gardens and Domain Trust, Sydney. http:// plantnet.rbgsyd.nsw.gov.au [consulta mayo 2019]

Plunkett, G. M. \& A. N. Nicolas. 2017 (15 sept 2016 on-line). Assessing Azorella (Apiaceae) and its allies: phylogenetics and a new classification. Brittonia 69: 31-61.

Spegazzini, C. L. 1899. Nova addenda ad Floram Patagonicam. Anales de la Sociedad Cientifica Argentina 48: 44-59.

Thiers, B. 2019 [permanentemente actualizado, consulta junio 2019]. Index Herbariorum: a global directory of public herbaria and associated staff. New York Botanical Garden's Virtual Herbarium. http://sweetgum.nybg.org/science/ih/

Turland, N. J., J. H. Wiersema, F. R. Barrie, W. Greuter, D. L. Hawksworth, P.S. Herendeen, S. Knapp, W.-H. Kusber, D. -Z. Li, K. Marhold, T.W. May, J. McNeill, A.M. Monro, J. Prado, M.J. Price \& G.F. Smith. 2018. International Code of Nomenclature for algae, fungi, and plants (Shenzhen Code) Glashütten: Koeltz Botanical Books, 240 pp.

Walpers, G. 1843. Cruciferas, Capparideas, Calycereas et Compositas, quas Meyenius in orbis circumnavigatione collegit, enumerat novasque describit G. Walpers, Dr. Nova acta physico-medica Academiae Caesareae LeopoldinoCarolinae Naturae Curiosum 19(1): 247-346.

Zech, J. C. 1995. Nomenclatural novelties on Mulinum (Apiaceae). Kurtziana 24: 192.

Zech, J. C. 1997. A new variety of Azorella diversifolia (Apiaceae) from Southern Chile. Madroño 44: 193-196. 